\title{
Effect of non surgical periodontal therapy on gingival crevicular fluid and serum visfatin concentration in periodontal health and disease
}

\author{
N.M. Raghavendra ${ }^{\mathrm{a}, *}$, A.R. Pradeep ${ }^{\mathrm{a}}$, Rahul Kathariya ${ }^{\mathrm{b}}$, Anuj Sharma ${ }^{\mathrm{a}}$, Nishanth S. Rao ${ }^{\mathrm{a}}$ and \\ Savitha B. Naik ${ }^{\mathrm{c}}$ \\ ${ }^{\mathrm{a}}$ Department of Periodontics, Government Dental College and Research Institute, Bangalore, India \\ ${ }^{\mathrm{b}}$ Department of Periodontics and Oral Implantology, Dr. DY Patil Dental College and Hospital, Pune, India \\ ${ }^{\mathrm{C}}$ Department of Conservative and Endodontics, Government Dental College and Research Institute, Bangalore, \\ India
}

\begin{abstract}
Visfatin is a pleiotropic mediator which acts as growth factor, cytokine, enzyme involved in energy including nicotinamide adenine dinucleotide metabolism and has been recently demonstrated to exert several pro-inflammatory functions. The purpose of this study is to evaluate the Visfatin concentration in gingival crevicular fluid (GCF) and serum in patients with chronic periodontitis, and to evaluate the effect of non-surgical periodontal therapy on the GCF and serum visfatin concentration. 30 subjects (age range: 25 to 52 years) were selected and divided into two groups based on the gingival index, probing depth, periodontal attachment level, and radiologic parameters (bone loss): group 1 (15 subjects with healthy periodontium), group 2 (15 subjects with chronic periodontitis), while, Group 2 patients after 8 weeks of the treatment (scaling and root planning, SRP) constituted group 3. GCF samples (by microcapillary pipettes) and serum samples (by venipuncture) were collected to estimate the levels of Visfatin using enzyme linked immunosorbent assay kit. The mean Visfatin concentration in GCF and serum was observed to be the highest in group 2 and lowest in group 1. While concentration in group 3 was similar to group 1 . The concentration of Visfatin in GCF and serum decreased after SRP. The Visfatin concentration in GCF and serum found to be highest in chronic periodontitis group and decreases after treatment. Hence Visfatin values can be considered as an "inflammatory marker" can be explored in future as a potential therapeutic target in the treatment of periodontal disease.
\end{abstract}

Keywords: Visfatin, gingivitis, chronic periodontitis, serum, adipokines

\section{Introduction}

Periodontitis is an inflammatory disease fundamentally initiated by chronic bacterial infection [1,2]. Current data suggest that a small group of predominantly gram-negative anaerobic or microaerophilic bacteria within the biofilm (dental plaque) are often associated with disease initiation and progression. The microbial

*Corresponding author: N.M. Raghavendra, Department of Periodontics, Govt. Dental College and Research Institute, Bangalore 560002, India. Fax: +91 080 26703176; E-mail: periodonticsgdcri@gmail.com. challenge consisting of antigens, lipopolysaccharides, and other virulence factors stimulates host responses. Host reactions to these infecting agents result in the release of inflammatory mediators (cells like neutrophils, monocyte/macrophages, $\mathrm{T}$ cells, mast cells, endothelial cells, fibroblasts etc.) including proinflammatory cytokines [Interleukin (IL)-1, IL-6, Tumour necrosis factor $\alpha$ (TNF- $\alpha)$ ] and prostaglandins (PGE2), which can promote extracellular matrix destruction (by matrix metalloproteinase; MMPs) in the periodontium and stimulate bone resorption [3]. Although these immune and inflammatory host reactions are essential for host defense against bacterial inflammation, excessive and 
prolonged reaction is harmful for the functional periodontal tissue.

The analysis of cytokine production levels has been also used as a tool for studying the local host response to a bacterial challenge. In particular, a large number of cytokines present in the gingival crevicular fluid (GCF) have been proposed as potentially useful diagnostic or prognostic markers of periodontal destruction [4, 5]. Various, proinflammatory cytokines like IL-1, IL-8, IL-6, TNF- $\alpha$; as well as anti-inflammatory cytokines like IL-4 and IL-10, were most commonly studied in the GCF, gingival tissue and serum of the healthy, gingivitis and chronic periodontitis patients [6-13]. Additionally, studies showed the pronounced effect of periodontal therapy (initial periodontal or surgical therapy) on levels of these cytokines [13-16]. This increased cytokines level in periodontitis may also exaggerate the systemic conditions, like atherosclerosis, diabetes, rheumatoid arthritis, preterm birth and respiratory conditions and recently, it has been considered as a risk factor for aforementioned conditions $[17,18]$.

Visfatin (also known as pre-B-cell colony-enhancing factor, PBEF) is a novel adipokine that is preferentially produced by visceral adipose tissue and has insulinmimetic actions [19]. It also functions as a proinflammatory adipocytokine that is secreted by neutrophils in response to inflammatory stimuli and upregulates the production of cytokines in the monocytes [20] suggesting a potential role in the pathogenesis of inflammatory disorders. Visfatin was found to be present in human peripheral blood monocytes [21], lymphocytes [22], macrophages [20,23] and dendritic cells [20]. The presence of Visfatin in a large variety of white blood cells and the tissue-bound macrophage suggests an important role for visfatin in the regulation of immune and defense functions [24].

Visfatin expression is up-regulated in a variety of acute and chronic inflammatory diseases including rheumatoid arthritis [25], sepsis [26], acute lung injury [27], inflammation, inflammatory bowel disease [20] and psoriasis [28] and plays a key role in the persistence of inflammation through its capacity to inhibit neutrophil apoptosis. Increased proinflammatory cytokines such as IL-6, IL-I $\beta$ and TNF- $\alpha$ can significantly increased visfatin expression [29].

The periodontal inflammation upregulates some proinflammatory cytokines such as IL-6, IL- $1 \beta$ which in turn can lead to high expression of visfatin in periodontal tissues. In our recent study [30] the Visfatin levels in GCF and serum in periodontal health and disease was estimated and results suggested that Vis- fatin levels increased progressively in GCF and serum with severity of periodontal disease. However, till date, Visfatin Concentration in GCF and serum in chronic periodontitis group of patients after periodontal therapy (scaling and root planning) has not been explored. Thus, in view of the aforementioned findings, this study was undertaken to know the effect of non-surgical periodontal therapy i.e. scaling and root planing (SRP) on GCF and serum Visfatin levels from subjects with chronic periodontitis and to analyze the correlation between GCF and serum Visfatin levels.

\section{Materials and method}

The study group consisted of 30 subjects, whose age (25-52 years) and sex (15 males and 15 females)matched, attended the outpatient section, Department of Periodontics, Government Dental College and Research Institute, Bangalore. Inclusion criteria included subjects within the age group 25-52 years with clinical signs of disease of their respective group, and who had at least 20 natural teeth. Patients with aggressive periodontitis, use of tobacco in any form, gross oral pathology, tumours, or any other systemic disease that can alter the course of periodontal disease, those who had taken medication affecting periodontal status or had received periodontal therapy in the preceding 6 months were excluded from the study. The Ethical Clearance was approved by Institutional ethical committee and Review board, Government Dental College $\&$ Research Institute, Bangalore, India. The protocol was clearly explained to all the patients and informed consent was obtained from all recruits. The study was conducted from mid September 2010 to January 2011. Each subject underwent a full-mouth periodontal probing and charting, and periapical radiographs were taken using the long-cone technique. Radiographic bone loss was recorded dichotomously (presence or absence) to differentiate patients with chronic periodontitis from other groups. Furthermore, no delineation was attempted within the chronic periodontitis group based on the extent of alveolar bone loss. Subjects were categorized into two groups based on the gingival index (GI) [31], probing depth (PD), periodontal attachment level (PAL), and radiographic evidence of bone loss. Group 1 (healthy) consisted of 15 subjects with clinically healthy periodontium, GI $=0$ (absence of clinical inflammation), PD $\leqslant 3 \mathrm{~mm}$, and PAL $=0$, with no evidence of bone loss on radiographs. Group 2 (chronic periodontitis) consisted of 15 subjects who had signs 
of clinical inflammation, GI $>1, \mathrm{PD} \geqslant 5 \mathrm{~mm}$, and PAL $\geqslant 3 \mathrm{~mm}$, with radiographic evidence of bone loss. Patients with chronic periodontitis (group 2) were treated with SRP, GCF and serum samples were collected 8 weeks after the treatment to constitute group 3 (the after-treatment group)

\subsection{Site selection and GCF fluid collection}

All the clinical and radiological examinations, group allocation and sampling site selection were performed by one examiner (ARP) and the samples were collected on the subsequent day by a second examiner (RNM). This was undertaken to prevent the contamination of GCF with blood associated with the probing of inflamed sites. Only one site per subject was selected as a sampling site in groups 2 and 3, whereas, in the healthy group, to ensure the collection of an adequate amount of GCF, multiple sites with absence of inflammation were sampled. In chronic periodontitis patients, sites with $>2 \mathrm{~mm}$ clinical attachment loss were identified, and the site showing the highest clinical attachment loss and signs of inflammation, along with radiographic confirmation of bone loss, was selected for sampling,

After making the subjects sit comfortably in an upright position on the dental chair, the selected test site was air dried and isolated with cotton rolls. Without touching the marginal gingiva, supragingival plaque was removed to avoid contamination and blocking of the microcapillary pipette. GCF was collected by placing white color-coded 1-5 $\mu \mathrm{l}$ calibrated volumetric microcapillary pipettes ${ }^{1}$ by placing the tip of the pipette extracrevicularly, until it gently touched the marginal gingiva, a standardized volume of $1 \mu \mathrm{l}$ unstimulated GCF was collected. The test sites which did not express standard volume $(1 \mu \mathrm{l})$ of GCF were excluded and micropipettes contaminated with blood and saliva was discarded. Periodontal treatment (SRP) was performed for periodontitis patients at the same appointment after GCF collection. After 8 weeks, GCF was collected from same site of the subjects in Group 2, and included in Group 3. For this 8-week period, subjects were called at one-week interval and plaque control measures were performed.

The GCF collected was immediately sealed with sterile aluminium foil and transferred to plastic vial and stored at $-70^{\circ} \mathrm{C}$ till the time of the assay.

\footnotetext{
${ }^{1}$ Sigma-Aldrich St. Louis, MO, USA.
}

\subsection{Collection of serum}

Two milliliters of blood was collected from the antecubital fossa by venipuncture using a 20 -gauge needle with $2 \mathrm{ml}$ syringe and immediately transferred to the laboratory. The blood sample was allowed to clot at room temperature and, after $1 \mathrm{~h}$, serum was separated from blood by centrifuging at $3000 \mathrm{~g}$ for $5 \mathrm{~min}$. The extracted serum was immediately transferred to a plastic vial and stored at $-70^{\circ} \mathrm{C}$ till the time of assay.

\subsection{Visfatin analysis}

The samples were assayed for Visfatin levels using commercially available enzyme-linked immunosorbent assay (ELISA) kit. ${ }^{2}$ Samples were analysed at the Department of Microbiology, Kempegowda Institute of Medical Sciences Bangalore, India.

All reagents were allowed to warm to room temperature $\left(18-25^{\circ} \mathrm{C}\right)$ before use. It is recommended that all standards and samples be run at least in duplicate. $100 \mu \mathrm{l}$ of anti-Visfatin antibodies were added to each well and incubated for 1.5 hours. The solution was discarded and the wells were washed 5 times with wash solution (200 $\mu \mathrm{l}$ each). Then $100 \mu \mathrm{l}$ of each standard, positive control and sample were added into appropriate wells. Wells were covered and incubated for 2.5 hours at room temperature. The solution was discarded and wells were washed 4 times with wash solution $(200 \mu \mathrm{l}$ each). $100 \mu \mathrm{l}$ of prepared HRP-Streptavidin solution was added to each well and incubated for 45 minutes at room temperature. The solution was discarded and wells were washed 5 times with wash solution $(200 \mu \mathrm{l}$ each). $100 \mu \mathrm{l}$ of TMB One-Step Substrate Reagent was added to each well and incubated for 30 minutes at room temperature in the dark. $50 \mu \mathrm{l}$ of Stop Solution was added to each well. Absorbance of the substrate colour reaction was read on ELISA reader ${ }^{3}$ using $450 \mathrm{~nm}$ as primary wavelength. The total Visfatin was determined in nanograms (ng), and the calculation of the concentration in each sample was performed by dividing the amount of Visfatin by the volume of sample (ng/ml).

\footnotetext{
${ }^{2}$ RayBio ${ }^{\circledR}$, Parkway Lane, GA, USA.

${ }^{3}$ Molecular Dynamics, Sunnyvale, CA, USA.
} 
Table 1

Descriptive statistics of the study population showing mean, standard deviation and range for the age, GI, PAL, PD, GCF and serum Visfatin concentrations

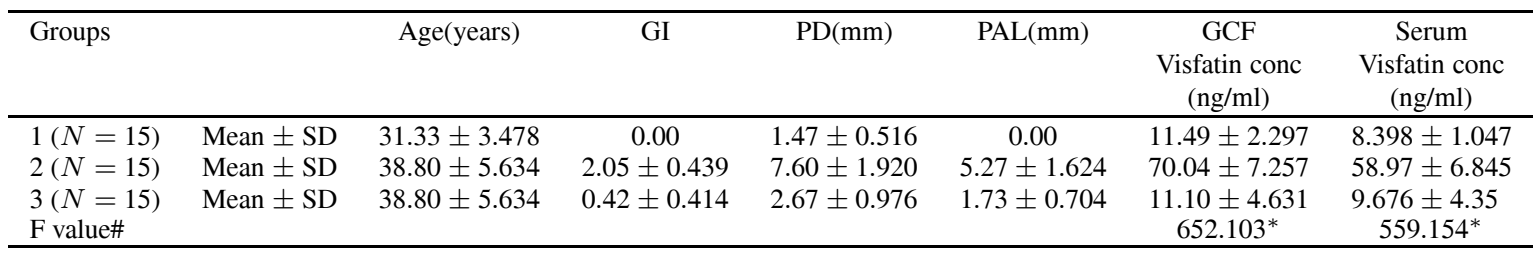

* Statistically significant at $p<0.05$.

\# ANOVA test.

GI: Gingival Index.

PD: Probing Depth.

PAL: Periodontal Attachment level.

\subsection{Statistical analysis}

All the data were analyzed using a software program. ${ }^{4}$ Based on the pilot study including five subjects in each group, the sample size was estimated at 15 subjects in each group to achieve power to detect a difference of 0.5 between the null hypotheses. Accordingly ANOVA tests were carried out for comparing the means of Visfatin concentration in different groups. Pair-wise comparison using scheff's test for GCF and serum Visfatin was carried out to explore, which pair or pairs differ significantly at 5\% level of significance. Paired t test was used to compare Visfatin concentrations in GCF and serum in groups 2 and 3.

\section{Results}

The Visfatin concentrations obtained for all the groups are shown in Table 1. The mean Visfatin concentration in GCF was observed to be the highest in group 2 while least in group 1. Group 3 mean Visfatin concentration in GCF was similar to group 1 . However, the mean Visfatin concentration in serum was found to be least in group 1 and group 3 and highest in group 2 .

To test the equality of mean between the groups ANOVA was carried out and hypothesis was rejected at 5\% significance (Table 1). Further multiple comparisons using Scheff's test was carried out to find out which pair or pairs differ significantly. On comparison of group 1 and 2, 2 and 3, the difference was statistically significant, both in GCF and serum $(p<0.001)$ (Table 2). When group 2 and group 3 were compared using paired ' $t$ ' test, the difference in the concentrations of Visfatin was statistically significant in both GCF $(t=$ $24.607)$ and serum $(t=21.695)$ at $p<0.001$.

\footnotetext{
${ }^{4}$ SPSS version 10.5 , SPSS Inc., Chicago, IL, USA.
}

Table 2

Pair-wise comparison using scheff's test for GCF and Serum Visfatin concentration

\begin{tabular}{lccccc}
\hline Groups & GI & $\begin{array}{l}\text { PPD } \\
(\mathrm{mm})\end{array}$ & $\begin{array}{l}\text { PAL } \\
(\mathrm{mm})\end{array}$ & $\begin{array}{l}\text { GCF } \\
\text { Visfatin } \\
\text { conc }\end{array}$ & $\begin{array}{l}\text { serum } \\
\text { Visfatin } \\
\text { conc }\end{array}$ \\
\hline 1 and 2 & $2.647^{*}$ & $6.133^{*}$ & $5.267^{*}$ & $58.55^{*}$ & $50.58^{*}$ \\
$p$-value & $<0.001$ & $<0.001$ & $<0.001$ & $<0.001$ & $<0.001$ \\
2 and 3 & $1.627^{*}$ & $4.933^{*}$ & $3.533^{*}$ & $58.938^{*}$ & $49.30^{*}$ \\
$p$-value & $<0.001$ & $<0.001$ & $<0.001$ & $<0.001$ & 0.008 \\
1 and 3 & $0.420^{*}$ & $1.200^{*}$ & $1.733^{*}$ & 0.385 & 1.278 \\
$p$-value & 0.008 & 0.47 & $<0.001$ & 0.979 & 0.0761 \\
\hline
\end{tabular}

* Statistically significant at $p<0.05$.

\section{Discussion}

The present study was designed to estimate the GCF and serum Visfatin concentration in periodontal health and disease as well as to evaluate correlation between GCF and serum visfatin levels before and after treatment.

Our present study comprised of three groups (healthy, chronic periodontitis and chronic periodontitis after treatment) to explore the role of SRP on GCF and serum Visfatin concentration, in addition to our recent study [30] where the Visfatin levels in GCF and serum in periodontal health and disease was estimated and results suggested that Visfatin levels increased progressively in GCF and serum as periodontal disease progressed. The additional group of periodontitis after treatment in our present study helped us to evaluate the effect of periodontal therapy on visfatin concentrations which can further confirm its role in periodontal disease.

In the current study design, the influence of age and gender on the Visfatin concentration was minimized by including an equal number of males and female in each group and selecting the subjects within the specified age group (25-52 years). The variability in the concentrations in each group could be due to different stages 
of the disease process at the time of collection of the GCF and serum samples.

In the present study, the extracrevicular (unstimulated) method of GCF collection using microcapillary pipettes was employed to ensure atraumatism, to obtain an undiluted sample of native GCF whose volume can be accurately assessed and also to avoid nonspecific attachment of the analyte to filter paper fibers [32,33]. The disadvantage of this method is the possibility of trauma to the marginal gingiva, and care was taken to avoid this during GCF collection. Furthermore, loss of GCF due to sticking of the sample to the capillary walls was avoided by flushing the capillary with a fixed amount of diluent, which was taken into account during the final calculations. In the present study, the use of commercially available, sensitive ELISA kit to quantify Visfatin from selected sites allowed us to avoid pooling of GCF samples from multiple sites or subjects.

The results of the present study indicated that concentration of Visfatin both in GCF and serum was highest in chronic periodontitis and decreased after periodontal therapy. Further the proportionate increase in levels from healthy to periodontitis groups in both GCF and serum confirms that Visfatin is released by predominant cells of periodontal disease activity. The concentration of Visfatin in the GCF was found to be greater than serum in all groups. This may be due to localized secretion of the Visfatin from the cells of periodontal tissue in response to inflammation. While in after treatment group (of chronic periodontitis) the mean concentration of Visfatin was similar to healthy subjects.

The wide range observed in the Visfatin levels in periodontitis and after treatment group could result, in part, from differences in disease activity and crevicular fluid flow as well as from the variations in the number of PMNs migrating into the crevice.

Visfatin and its mRNA expression are significantly increased in patients with inflammatory bowel disease [20], those with rheumatic arthritis [25] and in acute lung injury [27]. Visfatin enhance the expression of the adhesion and inflammatory proteins, ICAM-1, VCAM-1, E-Selectin [34]. Visfatin levels in chondrocytes increase in response to IL- $1 \beta$ and act in an autocrine /paracrine manner to trigger PGE2 synthesis [35]. Several lines of evidence demonstrate that adipokines, released by white adipose tissue, participate in a wide variety of physiologic and pathologic processes, including immunity and inflammation [36]. In the light of our results, we suggest that GCF and serum Visfatin concentration increases with severity of peri- odontal disease. Elevated serum Visfatin concentration is a known risk factor for cardiovascular disease [21]. This increased concentration of visfatin in periodontitis subjects, as seen in our study, indicates that the latter may raise the risk of developing cardiovascular disease. Therefore such increase in the serum concentration in chronic periodontitis patients may exaggerate or provoke such conditions in otherwise healthy individuals. Also, decreased level of such cytokine after periodontal therapy may reduce the risk for such conditions and so it is to be targeted for therapeutic potential in the treatment of periodontal diseases. Prospective studies with a larger sample size are essential to consider the rise in serum Visfatin as a risk factor for cardiovascular disease.

\section{Conclusion}

Based on the findings of the present study, increased Visfatin level both in the GCF and serum may suggest that its role in the pathogenesis of periodontal disease can be proposed.

Further longitudinal prospective studies involving a larger population are needed to confirm the findings of present study and to better understand the role of Visfatin in the pathogenesis. Also, addressing the clinical implication and pathological mechanism of Visfatin in periodontal disease progression are warranted.

\section{Acknowledgement}

The present study was partly funded by Colgate research grant, Colgate Palmolive India limited, Mumbai, India.

\section{References}

[1] Listgarten MA, Pathogenesis of periodontitis, J Clin Periodontal 13 (1986), 418-30.

[2] Jotwani R, Cutler CW, Adult periodontitis-specific bacterial infection or chronic inflammation? J Med Microbiol 47 (1998), 187-8.

[3] Suda T, Takahashi N, Udagawa N, Jimi E, Gillespie MT, Martin TJ, Modulation of osteoclast differentiation and function by the new members of the tumour necrosis factor receptor and ligand families, Endocr Rev 20 (1999), 345-57.

[4] Birkedal-Hansen H, Role of cytokines and inflammatory mediators in tissue destruction, J Periodontal Res 28 (1993), 500-510.

[5] Genco R, Host responses in periodontal diseases: current concepts, J Periodontol 63 (1992), 338-355. 
[6] Kamma J, Mombelli A, Tsinidou K, Vasdekis V, Giannopoulou $\mathrm{C}$, Cytokines in gingival crevicular fluid of adolescents and young adults, Oral Microbiol Immunol 24 (2009), 7-10.

[7] Kato T, Akiyama H, Sugano N, et al, Study of Granzyme A and Interleukin 2 Expression in Periodontitis, Journal of the Japanese Association of Periodontology 36 (1994), 592-598.

[8] Stashenko P, Fujiyoshi P Obernesser MS, Prostak L, Haffajee AD, Socransky SS, Levels of interleukin1in tissue from sites of active periodontal disease, J Clin Periodontol 18 (1991), 548-54.

[9] Fujihashi K, Kono Y, Beagley KW, et al, Cytokines and periodontal disease: Immunopathological role of interleukins for B cell responses in chronic inflamed gingival tissues, $J$ Periodontol 64 (1993), 400-6.

[10] Okada H, Murakami, S, Cytokine expression in periodontal health and disease, Crit Rev Oral Biol Med 9 (1998), 248-66.

[11] Shapira L, Van Dyke TE, and Hart TC, A localized absence of IL-4 triggers periodontal disease activity: a novel hypothesis, Medical hypotheses 39 (1992), 319-22.

[12] Yun F, Firkova EI, Xun H, Jun-Qi L, Effects of surgical periodontal therapy on serum levels of TNF-alpha in patients with chronic periodontitis, Folia Med (Plovdiv) 49 (2007), 37-40.

[13] Blach A, Franek E, Witula A, et al, The influence of chronic periodontitis on serum TNF-alpha, IL-6 and hs-CRP concentrations, and function of graft and survival of kidney transplant recipients, Clin Transplant 23 (2009), 213-9.

[14] Hussain Bokhari SA, Khan AA, Tatakis DN, Azhar M, Hanif M, Izhar M, Non-surgical periodontal therapy lowers serum inflammatory markers: a pilot study, J Periodontol 80 (2009), 1574-80.

[15] Dağ A, Firat ET, Arikan S, Kadiroğlu AK, Kaplan A, The effect of periodontal therapy on serum TNF-alpha and HbA1c levels in type 2 diabetic patients, Aust Dent J 54 (2009), 17-22.

[16] Talbert J, Elter J, Jared HL, Offenbacher S, Southerland J, Wilder RS, The effect of periodontal therapy on TNF-alpha, IL-6 and metabolic control in type 2 diabetics, J Dent Hyg $\mathbf{8 0}$ (2006), 7.

[17] Mealey BL, Influence of periodontal infections on systemic health, Periodontol 200021 (1999), 197.

[18] Page RC, Beck JD, Risk assessment for periodontal diseases, Int Dent J 47 (1997), 61.

[19] Fukuhara A, Matsuda M, Nishizawa M, et al, Visfatin: a protein secreted by visceral fat that mimics the effect of insulin, Science 307 (2005), 426-430.

[20] Moschen AR, Kaser A, Enrich B, et al, Visfatin adipocytokine with inflammatory and immunomodulating properties, $\mathrm{J}$ Immunol 178 (2007), 1748-1758.

[21] Dahl TB, Yndestad A, Skjelland M et al, Increased expression of Visfatin in macrophages of human unstable plaque destabilization, Circulation 115 (2007), 972-980.

[22] Samal B, Sun Y, Stearns G, Xie C, Suggs S, McNiece I, Cloning and charecterization of the cDNA encoding a novel human pre-B cell colony enhancing factor, Mol Cell Biol 14 (1994), 1431-1437.

[23] Curat CA, Wegner V, Sengenes C, et al, Macrophages in human visceral adipose tissue: increased accumulation in obesity and a source of resistin and visfatin, Diabetologia 49 (2006), 744-747.

[24] Sethi JK, Vidal-Puig A, Visfatin: the missing link between intra-abdominal obesity and diabetes? Trends Mol Med 11 (2005), 344-347.

25] Nowell MA, Richards PJ, Fielding CA, et al, Regulation of pre-B cell colony-enhancing factor by STAT-3-dependent interleukin-6 trans-signaling: implications in the pathogenesis of rheumatoid arthritis, Arthritis Rheum 54 (2006), 20842095.

[26] Jia SH, Li Y, Parodo J, et al, Pre-B cell colony-enhancing factor inhibits neutrophil apoptosis in experimental inflammation and clinical sepsis, J Clin Invest 113 (2004), 1318-1327.

[27] Ye SQ, Simon BA, Maloney JP, et al, Pre-B-cell colonyenhancing factor as a potential novel biomarker in acute lung injury, Am J Respir Crit Care Med 171 (2005), 361-370.

[28] Koczan D, Guthke R, Thiesen HJ, et al, Gene expression profiling of peripheral blood mononuclear leukocytes from psoriasis patients identifies new immune regulatory molecules, Eur J Dermatol 15 (2005), 251-257.

[29] Ognjanovic S, Bao S, Yamamoto SY, Garibay-Tupas J, Samal B, Bryant-Greenwood GD, Genomic organization of the gene coding for human pre-B-cell colony enhancing factor and expression in human fetal membranes, J Mol Endocrinol 26 (2001), 107-117.

[30] Pradeep AR, Raghavendra NM, MV Ramchandra Prasad, Patel SP, Kathariya R, Sharma A, Gingival crevicular fluid and serum Visfatin concentration: their relationship in periodontal health and disease, J Periodontol. 2011 Feb 10. [Epub ahead of print]

[31] Loe H, Silness J, Periodontal disease in pregnancy. I. Prevalence and severity, Acta Odontol Scand. 21 (1963), 533-551.

[32] Sueda T, Bang J, Cimasoni G, Collection of gingival fluid for quantitative analysis, J Dent Res 48 (1969), 159.

[33] Pradeep AR, Daisy H, Hadge P, Garg G, Thorat M, Correlation of gingival crevicular fluid interleukin-18 and monocyte chemoattractant protein-1 levels in periodontal health and disease, J Periodontol 80 (2009), 1454-1461.

[34] Su-Ryun K, Yun-Hee B, Soo-Kyung B, et al, Visfatin enhances ICAM-1 and VCAM-1 expression through ROS-dependent NF-kB activation in endothelial cells, Biochim Biophys Acta 1783 (2008), 886-895.

[35] Marjolaine G, Francis B, Colette S, et al, Crucial role of Visfatin in matrix degradation and prostaglandin $\mathrm{E}_{2}$ synthesis in chondrocytes, Arthritis Rheum 58 (2008), 1399-1409.

[36] Alexander R, Moschen Arthur K, Babara E, et al, Visfatin, an adipocytokine with proinflammatory and immunomodulating properties, J immunol 178 (2010), 1748-1758. 


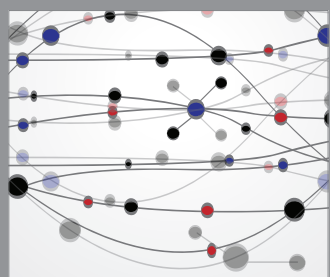

The Scientific World Journal
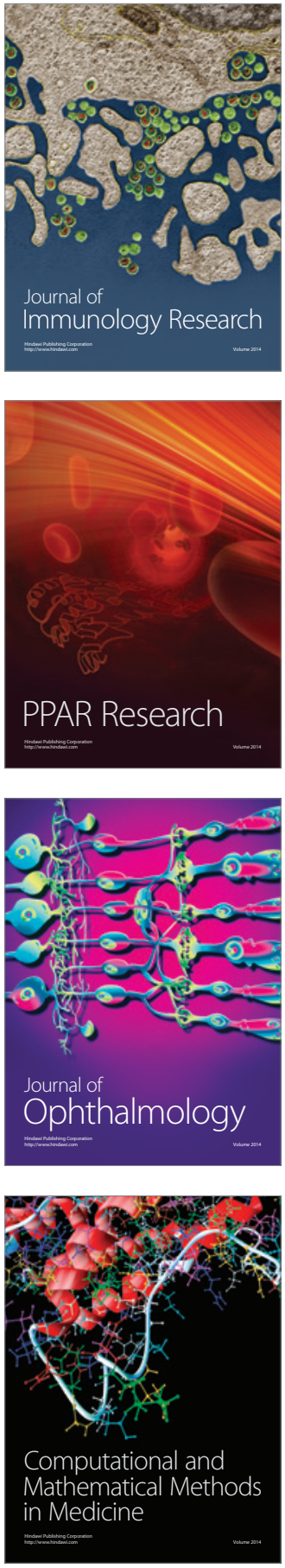

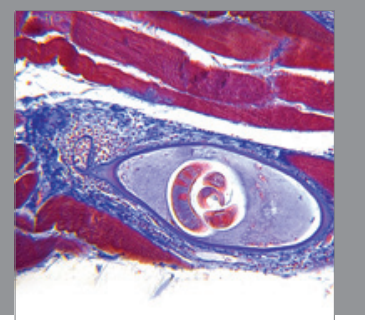

Gastroenterology

Research and Practice
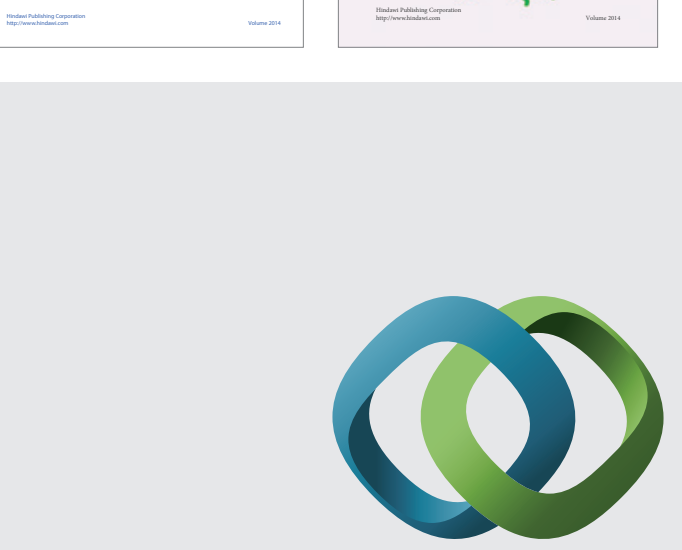

\section{Hindawi}

Submit your manuscripts at

http://www.hindawi.com
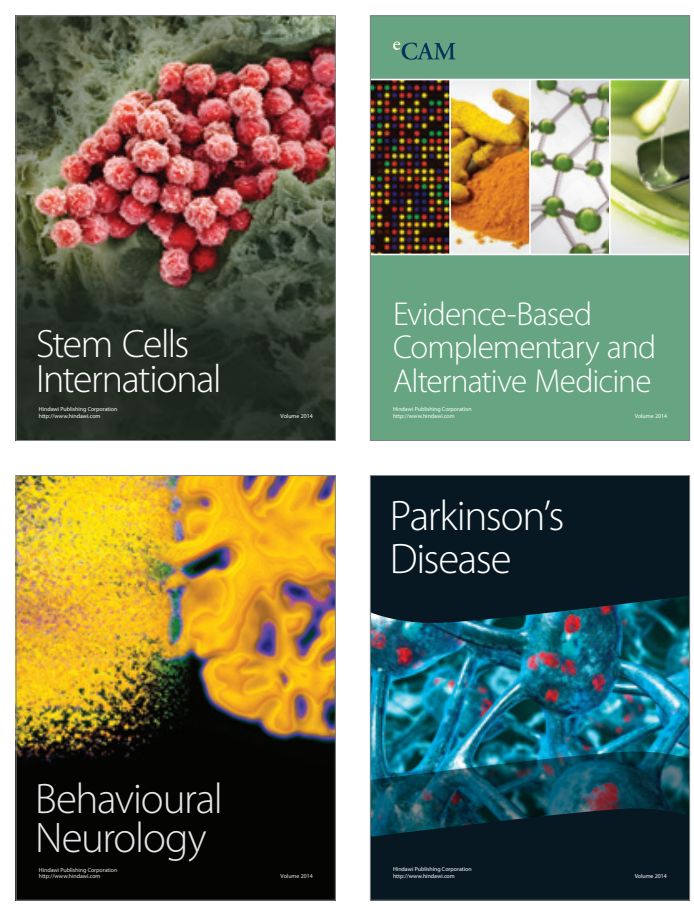

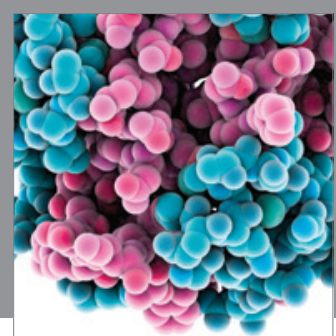

Journal of
Diabetes Research

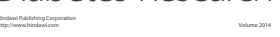

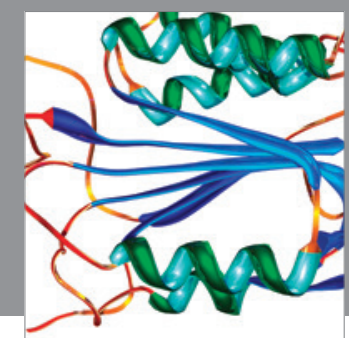

Disease Markers
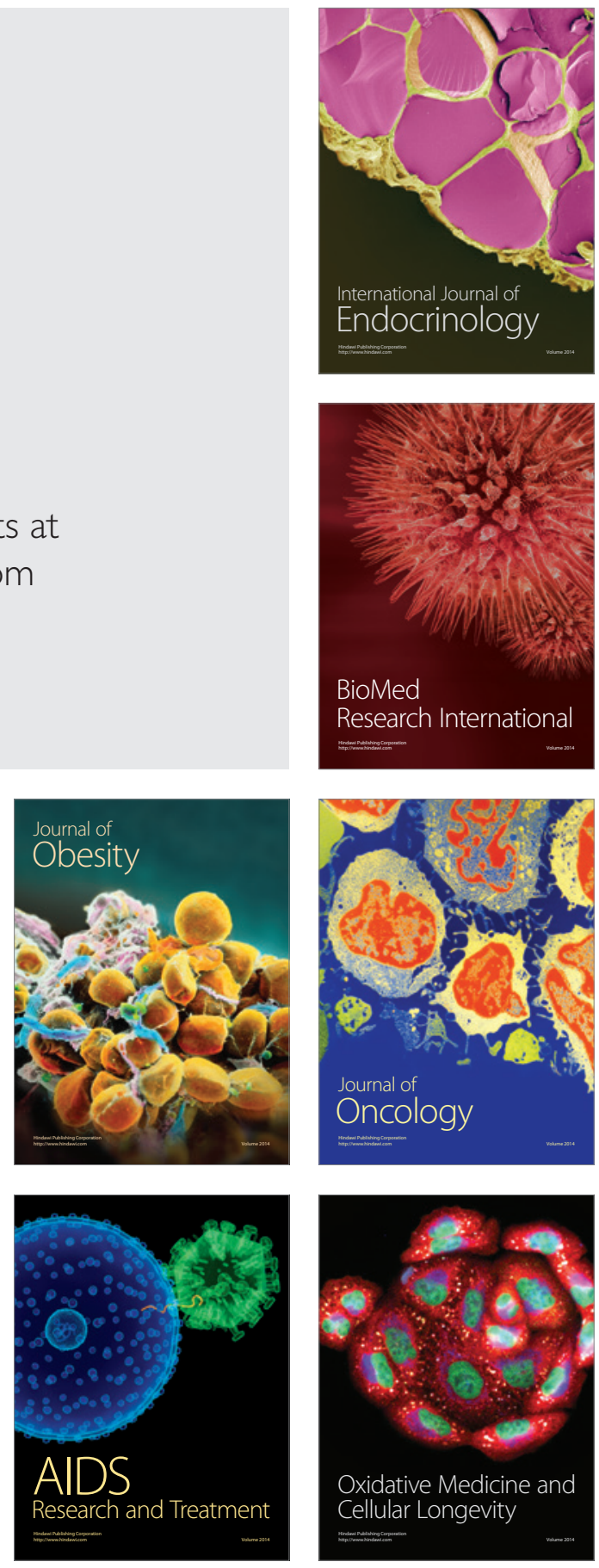